\title{
Male Breast Carcinoma: Correlation of ER, PR, Ki-67, Her2-Neu, and p53 with Treatment and Survival, a Study of 65 Cases
}

Jessica Wang-Rodriguez, M.D., Keith Cross, Scott Gallagher, M.D., Marcia Djahanban, Janet M. Armstrong, Noel Wiedner, M.D., David H. Shapiro, M.D.

Department of Pathology, University of California, San Diego (JWR, NW, KC) and Pathology and Laboratory Medicine Services, VA San Diego Healthcare System (JWR, MD, JA), San Diego, California; Department of Surgery, University of South Florida College of Medicine (SC, DS), Tampa, Florida; and Bay Pine VA Medical Center (DS), Bay Pine, Florida

Male breast cancer is rare, and experience of it in any single institution is limited. Our current understanding regarding its biology, natural history, and treatment strategies has been extrapolated from its female counterpart. The aim of this study is to evaluate the expression patterns of estrogen receptor (ER), progesterone receptor (PR), MiB1 (Ki67), Her2/neu (c-erbB2), and p53 and to correlate them with the prognosis, presentation, staging, management, and survival/outcome in male breast carcinoma identified through the Veterans Administration nationwide cancer registry. Sixty-five cases of male breast cancer were reviewed for classification. Tumor blocks were requested from each institution for immunohistochemical staining and evaluation of ER, PR, p53, Her2-neu, and MiB1. Seventeen ageand disease-matched male veteran patients with breast gynecomastia were used as controls. Traditional prognostic data were collected for comparison with female breast cancers (i.e., age, lymph node status, clinical staging, tumor size, histological grade, and disease-free and overall survival). Male breast carcinoma had worse disease-free survival than controls $(P=.03)$. The clinical stage regardless of tumor size or lymph node metastasis was the single most significant prognostic factor $(P<$ .0001). ER-positive patients appeared to have a better survival than did ER-negative patients $(P=.03$, univariate; $P$ not significant in multivariate) and did not benefit from treatment with tamoxifen $(P=$

Copyright (C) 2002 by The United States and Canadian Academy of Pathology, Inc.

VOL. 15, NO. 8, P. 853, 2002 Printed in the U.S.A.

Date of acceptance: April 5, 2002.

Sponsored by American Cancer Society Grant IRG 70-002-29.

Address reprint requests to: Jessica Wang-Rodriguez, M.D., University of California, San Diego, School of Medicine, Department of Pathology 0612, 9500 Gilman Drive, La Jolla, CA 92093; e-mail: jwrodriguez@ucsd.edu; fax: 858-552-4370.

DOI: 10.1097/01.MP.0000022251.61944.1D
.0027, univariate; $P=.42$, multivariate). $\mathrm{MiB1}$ and PR expressions did not correlate with treatment or survival, and p53 was associated with shorter disease free survival $(P=.07$, univariate; $P=.047$, multivariate). Stage for stage, Her2-neu was associated with shorter disease-free survival $(P<.0001)$ and correlated with positive lymph nodes $(P=.08)$. Surgery alone versus surgery with adjuvant treatments (chemotherapy, radiotherapy, tamoxifen, or combination) did not show any survival difference. Adjuvant therapy seemed to be associated with worse outcome. In the Veterans Administration hospital setting, the clinical stage and the expressions of p53 and Her2-neu in male breast carcinoma may be prognostically useful markers in guiding future treatment in prospective studies, whereas ER, PR, and MiB1 expressions are of limited value.

KEY WORDS: Estrogen receptor, Her2-neu, Hormone receptors, Immunohistochemistry, Ki-67, Male breast carcinoma, Mib-1, p53, Progesterone receptor.

Mod Pathol 2002;15(8):853-861

Male breast carcinoma is rare, with an estimated 1,000 to 1,400 new cases per year (1). The tumor phenotypic alterations are not well studied, and experience is mainly inferred from that of female breast cancer. Although both diseases have similarities, there are notable differences in risk factors, prognosis, and survival. Reported differences between male and female breast carcinoma have been noted, and male breast carcinoma has a tendency to present at higher clinical stages and with more lymph node metastases (2-4). Many molecular markers are available for the better understanding of female breast cancer tumorigenesis and disease progression and possibly to guide treatment. How- 
ever, few studies have been performed on the male breast counterpart. The clinical and pathological understanding of breast cancer in male patients is limited because the incidence of this disease is quite low in any single institution $(2,3,5-7)$.

The aim of this study is to examine the prognostic role of MiB1 (Ki-67, proliferation antigen), Her-2/ neu oncogene, and p53 protein mutation, as well as traditional prognostic markers of estrogen receptor (ER) and progesterone receptor (PR) expression in male breast carcinomas from archived tumor blocks obtained from multiple Veterans Administration (VA) hospitals using immunohistochemical methods. The results will be correlated with the patient's clinical stage and disease-free and overall survival.

\section{MATERIALS AND METHODS}

\section{Patient Clinical Data}

The Veterans Affairs database provides a unique source of a large number of male patients available for analysis. The patient's records are available in a standardized format through a single accessible computer system. Information was obtained from the Department of Veteran's Affairs (DVA) Cancer Registry, and Patient Treatment File (PTF), using the ICD-CM diagnostic codes 611.1 (hypertrophy of the male breast), 611.72 (breast lump or mass), 217 (benign neoplasm male breast), 175.9 (malignant neoplasm male breast). Individual VA tumor registrars and medical record departments that listed patients treated with these diagnoses/were contacted and the records requested. The Beneficiary Identification and Record Location System were used to obtain data on mortality after discharge. These databases contained from $80-90 \%$ of the recorded veterans' deaths. If an additional check of the data compared with the data in the U.S. Social Security Administration failed to indicate death, then the patient was recorded as alive at their last known follow-up date.

Charts of cases of male breast cancer identified over a decade were obtained from the records departments of the VA hospitals. Analysis included demographic data, history of the onset, and presentation in the breast including a mass, area of tenderness, or nipple change or enlargement of the breast. Physical findings were categorized for the location and size of the lesion and the nipple areolar complex and the presence of regional adenopathy. Comorbidities as well as other systemic physical findings of significance were noted. An additional breast tissue control of 17 noncancerous male veteran patients matched for age and underlying medical diseases with diagnosis of gynecomastia was used for comparison of the stains.
Histology and Immunohistochemistry:

Original pathology reports were assessed to include histology, grade, nuclear analysis, and receptor status. Traditional prognostic data were collected to compare with that of female breast cancers (i.e., age, lymph node status, clinical and pathological staging, tumor size, and histological grade). Adjuvant treatment (chemotherapy, radiation, or tamoxifen) was noted. Tumor blocks were requested from each institution for immunohistochemical staining for ERs and PRs (DAKO, Carpinteria, CA) as well as recently described breast cancer prognostic markers such as p53 (Novocastra Laboratory Ltd., Newcastle, UK), Her2-Neu (DAKO), and Ki-67 (MiB1-Innovex, Inc., Parsippany, NJ), using established antigen retrieval method. Pathology slides were reviewed independently and concurrently by two pathologists (JWR and NW) for nuclear grading according to the modified Bloom and Richardson system (8), classification of tumors, and evaluation of the immunohistochemical stains. Discrepancies were resolved after concurrent review of the cases in question. When there were discrepancies of the nuclear grade, histologic type, or ER and PR receptor status from the original pathological report, the new histological grade or staining results were recorded for our data analysis. The patient medical records and tissue blocks contained original identification numbers and therefore warranted the process of institutional review for use of human records and tissue; the study was approved by the institutional review board at University of California, San Diego.

\section{Scoring}

ER and PR nuclear stains were categorized as positive or negative. The positive staining was established as $>50 \%$ of the cell nuclei stained. p53 nuclear stains were scored as positive for strong, uniform staining of the tumor nuclei, as indeterminate for weak or focal nuclear staining, and as negative for no staining. Her2-Neu expression was graded as positive when strong, membranous stains surrounded the entire cytoplasmic circumference of the tumor cell (equivalent to the FDA-approved DAKO HercepTest $2+$ and $3+$ ), indeterminate for partial membranous staining $(1+)$, and negative for indistinct or no staining of the tumor cells $(0+; 9)$. The expression characteristics were modified to three variables to improve statistical power because Her2-Neu-positive cases were too few in our cohort. MiB1 was expressed as a percentage per 1000 tumor cells. Appropriate positive controls from female breast tumors were used for each antibody. 
Statistical Analysis:

Statistical analysis was applied to conclusions drawn from study of the database itself, as well as comparison to reports in the literature of male breast cancer in the general population, and of female breast cancer. All statistical analyses used StatView Version 5.0.1 (SAS Institute, Inc., Cary, $\mathrm{NC}$ ). Comparison of categorical data was done with either $\chi^{2}$ or Fisher's exact test depending on the sample size. Comparison of numerical data was done with the $t$ test. Survival analysis was performed with the Kaplan-Meier curves and significance determined by log-rank test (KM-LR) for selected groups of patients. The parameters significant in the log-rank univariate test were tested in the stepwise regression procedures. The multivariate analysis was performed using the Cox's proportional hazard models. Death due to tumor and tumor recurrence was considered failures. Those patients who died of other causes or were alive without disease were censored at the time of death or were lost to follow-up.

\section{RESULTS}

A total of 241 patients were identified in the VA tumor registry database, but only 65 patients had complete follow-up history and pathology blocks available in the archives for review. The patients were diagnosed between 1990-2000, with a median follow-up of 36.7 months (range $=1-134 \mathrm{mo}$ ). Seventeen cases of age-matched male patients with benign gynecomastia were studied as control. The average age for the male breast cancer patients was $68.2 \pm 12$ years (range, 35 to $89 \mathrm{y}$ ) and for control patients, $66 \pm 8$ years (range, 52 to $81 \mathrm{y} ; P=\mathrm{ns}$ ). The results of the pathological review by the authors (JWR and/or NW) as well as other patient demographic data were summarized in Table 1. The overall survival for male breast cancer patients was significantly worse than that for the control male patients $(36 \pm 27$ mo for MBC versus $51 \pm 36$ mo for control, $P=.03$, Kaplan-Meier and log rank; Fig. 1 and Table 2). There was no difference in patients' smoking status (30\% MBC versus $45 \%$ control) and other background medical histories between the MBC and control patients. Only two MBC patients had documented family history of breast cancer (one mother and one sister). Tumors that contained ductal carcinoma in situ (DCIS) did not confer survival advantage $(30.3 \pm 23.4$ mo without DCIS versus $32.5 \pm 8.4$ mo with DCIS, $P=.8$, Table 2). The average tumor size was $2.9 \pm 1.9 \mathrm{~cm}$ (range, 0.3 to $10 \mathrm{~cm}$ ). There was no survival difference in tumor size, histological grade, or lymph node metastasis at the time of diagnosis in this cohort (Table 2). There was a trend of increased
TABLE 1. Patient Demographics of the Male Breast Carcinoma (MBC) Cohort

\begin{tabular}{lrr}
\hline \multicolumn{1}{c}{ MBC Patients } & $N(n=65)$ & Percentage \\
\hline Histological types & & \\
Infiltrating ductal carcinoma, NOS & 44 & 67.7 \\
DCIS & 2 & 3.1 \\
Adenocarcinoma, NOS & 1 & 1.5 \\
Invasive papillary carcinoma & 1 & 1.5 \\
Mucinous carcinoma & 2 & 3.1 \\
DCIS and infiltrating ductal carcinoma & 15 & 23.1 \\
Nuclear grades & & \\
Grade 1 & 9 & 13.8 \\
Grade 2 & 31 & 47.7 \\
Grade 3 & 25 & 38.5 \\
Lymph node metastasis & & \\
Yes & 31 & 47.7 \\
No & 34 & 52.3 \\
TNM stages & & \\
0 & 1 & 1.5 \\
1 & 17 & 26.2 \\
2 & 29 & 44.6 \\
3 & 11 & 16.9 \\
4 & 7 & 10.8 \\
Patient ethnicity & & \\
Caucasian & 41 & 63.1 \\
African-American & 17 & 26.2 \\
Hispanic/Latino & 4 & 6.2 \\
Unknown & 3 & \\
\hline & & \\
DCIS, ductal carcinoma & & \\
\hline
\end{tabular}

DCIS, ductal carcinoma in situ; NOS, not otherwise specified.

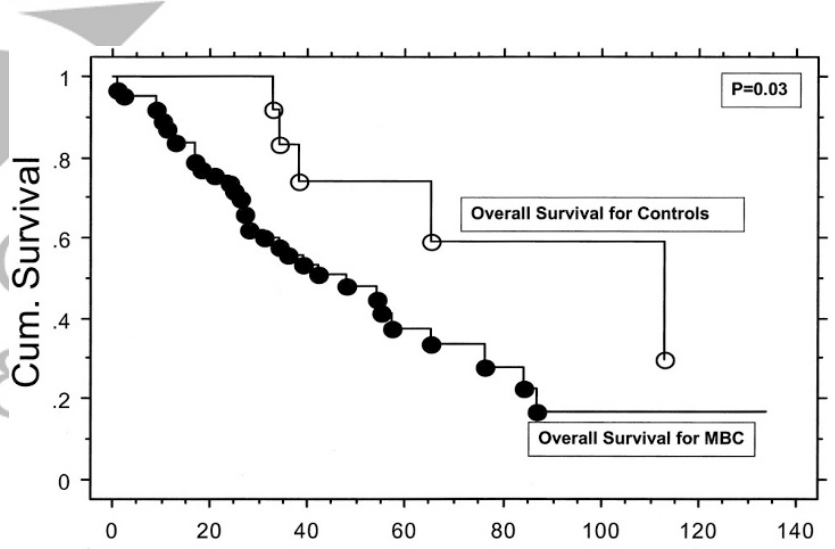

Time in Months

FIGURE 1. The overall survival for patients with male breast carcinoma (MBC) versus control patients with benign gynecomastia. The survival is significantly worse in patients with breast carcinoma $(P$ $=.03)$.

tumor grade that was associated with a higher number of positive lymph nodes $(P=.09)$. However, advanced tumor stage (American Joint Committee on Cancer) was strongly associated with shortened disease-free survival (Kaplan-Meier and $\log$ rank $P<.0001$; Fig. 2), implying that tumor metastasis was significantly associated with adverse survival.

The results of the immunohistochemical analysis of the antibodies, ER, PR, MiB1, Her2-neu, and p53 were summarized in Table 3. Stains for ER, PR, $\mathrm{MiB1}$, and p53 showed distinct nuclear staining in tumor cells, whereas Her2-neu stains were characteristically membranous (Fig. 3). The number of 
TABLE 2. Months of Survival By Tumor Characteristics

\begin{tabular}{lcc}
\hline \multicolumn{1}{c}{ Characteristic } & Months & $P$ Value \\
\hline MBC patients & $36 \pm 27$ & - \\
Control patients & $51 \pm 36$ & 0.03 \\
MBC without DCIS & $30.3 \pm 23.4$ & - \\
MBC with DCIS & $32.5 \pm 8.4$ & 0.8 \\
Histological Grade 1 & $38.5 \pm 20$ & - \\
Grade 2 & $33.1 \pm 27.9$ & - \\
Grade 3 & $28.1 \pm 18.4$ & 0.7 \\
Negative lymph node & $30.2 \pm 17.4$ & - \\
Positive lymph node & $38.1 \pm 29.3$ & 0.43
\end{tabular}

MBC patients had fewer months of survival than did control patients. DCIS, histological grade, and lymph node metastasis were not significant in overall survival. DCIS, ductal carcinoma in situ; NOS, not otherwise specified; MBC, male breast carcinoma.

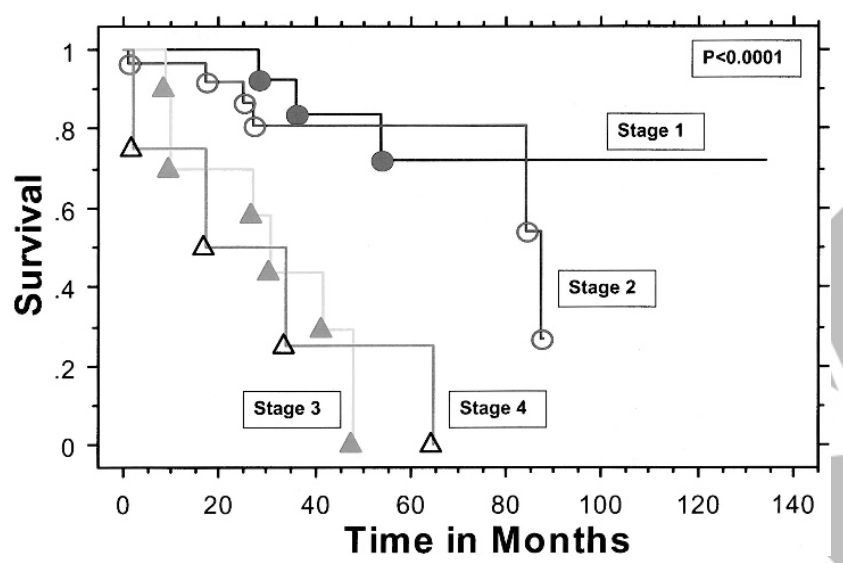

FIGURE 2. The disease-free survival of MBC patients in four clinical stages. There is a statistically significant difference in survival with regard to stages $(P<.0001)$.

ER- and PR-positive tumors was not significantly different from control. The mean percentage of MiB1 in tumors was $10.6 \%$ and $3.5 \%$ in control cases, and no control cases had a MiB1 score of $>10.6 \%(P=.0088)$. p53 was expressed more in tumors than in controls $(P=.0087)$. The Her2-neu positive rate was $9 \%$, and all of the control cases were Her2-neu negative $(P=.10)$.

Patients with ER-positive tumors appeared to have a better survival ( $n=62, P=.03, \mathrm{KM}-\mathrm{LR})$ than did patients who were ER negative $(n=3)$ in the univariate analysis but not in multivariate analysis. In Table 4, ER did not correlate with tumor grade, lymph node positivity, or clinical stages at diagnosis. PR-positive tumors correlated with high-grade tumors $(P=.04$; Table 4$)$. However, PR did not offer any differences in disease-free survival. Higher MiB1 percentage $(>10.6 \%)$ did not appear to associate with positive lymph nodes, tumor grades, stage, or survival (Table 4). There was no correlation between p53 and tumor grade, lymph node metastasis, or stage in MBC patients (Table 4), but there was a suggestion that p53+ tumors had shorter disease-free survival $(P=.07$; Fig. 4$)$, and this correlation became confirmatory when stratified by stage in multivariate analysis $(P=.047)$.
Her2-neu-positive patients tended to have positive lymph nodes $(P=.08$; Table 4$)$ and a higher number of positive lymph nodes $(P=.04)$. Worse disease-free survival in Her2-neu-positive tumors was observed in both early Stage I/II and late Stage III/IV patients $(P<.0001$; Fig. 5). Multivariate analysis performed on stage, p53, and Her2-neu continued to show that advanced stage $(P=.0001)$ and positive Her2-neu in Stage III/IV patients $(P=.04$, Fig. 6) were independent adverse prognostic factors, but p53 was not $(P=.4)$.

Ninety-seven percent of the patients underwent surgery $(n=62)$. The types of surgery included simple mastectomy $(n=9)$, excisional biopsy only $(n=2)$, modified radical mastectomy $(n=41)$, and radical mastectomy $(n=10)$. Twenty-nine percent of the patients chose to have surgery as the only therapy, whereas $71 \%$ of the patients had surgery and adjuvant therapies. Adjuvant therapies included chemotherapy ( $n=15)$, hormonal therapy (tamoxifen, $n=27)$, radiotherapy $(n=17)$, or a combination of the above $(n=15)$. There was no consistency or specific criteria in adjuvant therapy in this cohort, and the options were decided on either by the patients' physicians or patients themselves. There was no difference in survival in patients receiving adjuvant therapy versus patients who had only surgery (mean survival $=39 \pm 30 \mathrm{mo}$ for surgery alone versus $30 \pm 24$ mo in surgery plus adjuvant therapy, $P=.29$ ). This finding was confirmed by the log rank test.

When comparing the survival of various antigenpositive tumors and their treatment, we found that the ER-positive patients who were on hormonal (tamoxifen) therapy had a less favorable survival ( $P$ $=.0027$, univariate analysis), but this significance was diminished in multivariate analysis $(P=.42)$. When comparing the patients' stages with regard to adjuvant therapy, a worse disease-free survival was seen in patients who received radiotherapy or chemotherapy than in patients who did not receive either therapy ( $P=.0002$ and 0.0003 , respectively). However, the statistical power was markedly diminished because of small sample size. Other markers were not significant in offering survival benefit in patients who received adjuvant therapy.

\section{DISCUSSION}

Male breast carcinoma usually presents as a painless, firm subareolar mass or a mass in the upper outer quadrant of the breast. Some of the risk factors implicated include diet (10), alcohol intake, obesity (11), tobacco abuse, and conditions associated with increased estrogen or decreased androgen, medications, or radiation exposure (12). Similar to the case of breast carcinoma in females, a family history of breast cancer in men or women 


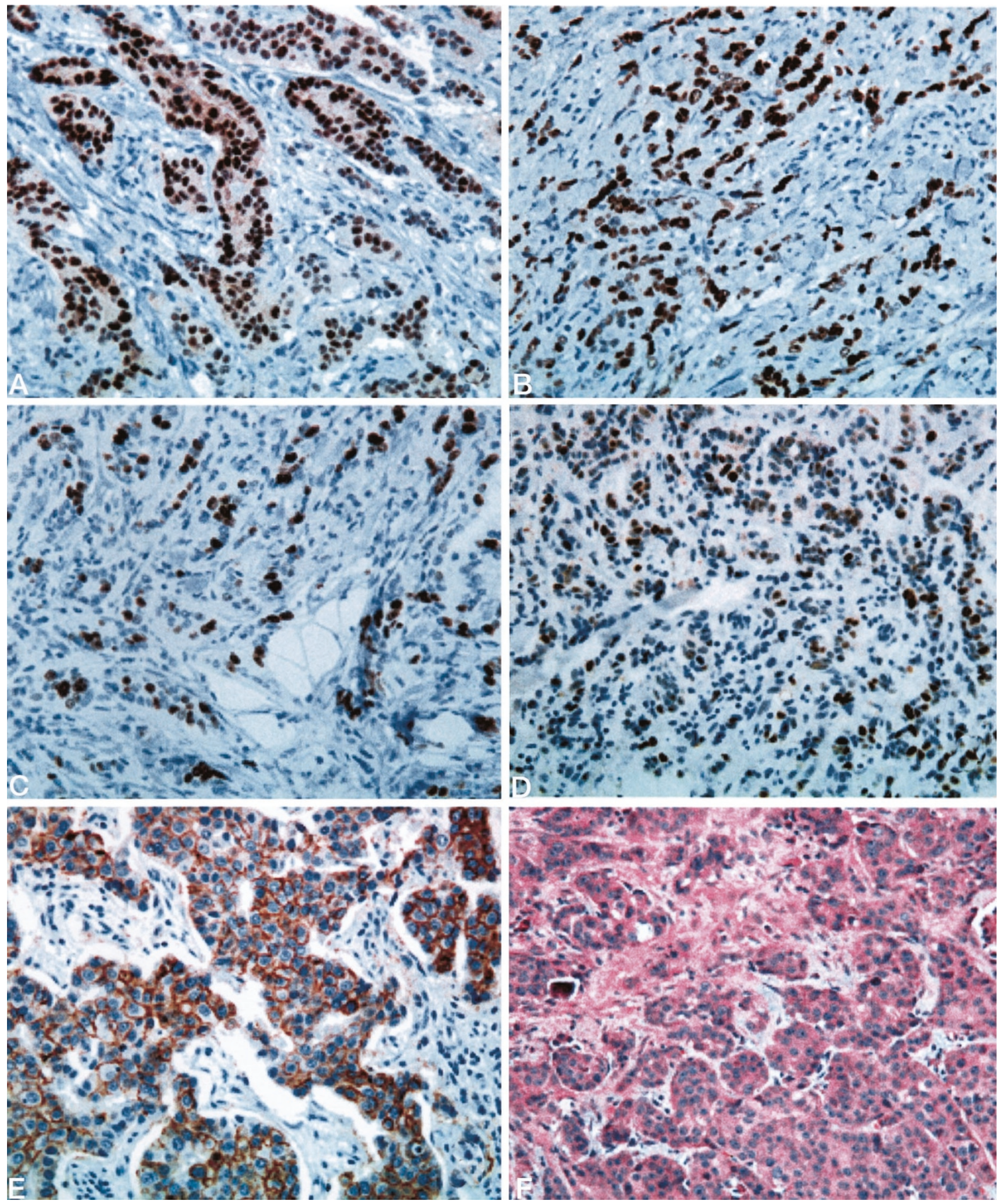

FIGURE 3. Composite figures of immunohistochemical stains of ER (A), PR (B), MiB (C), p53 (D), and Her2-neu (E). Stains for ER, PR, MiB, p53 are nuclear, whereas Her2-neu is membranous. Her2-neu in $\mathbf{E}$ is scored as positive (DAKO criteria $3+$ ). A hematoxylin and eosin stain of a representative infiltrating ductal carcinoma is also included (F). All photographs, 200×.

increases the risk for breast cancer (13). One study reported that low-stage patients had higher grade tumors and shortened survival (14). However, in general, the disease behaves like female breast can- cer, following similar patterns of local invasion and metastasis.

The chosen patient population was somewhat different than the general population in their mili- 
TABLE 3. Immunohistochemical Stains for the Male Breast Carcinoma (MBC) Tumor Versus Control (CTR, gynecomastia)

\begin{tabular}{|c|c|c|c|c|c|c|}
\hline \multirow{2}{*}{ Stain } & \multicolumn{3}{|c|}{ MBC Patients $(N=65)$} & \multicolumn{2}{|c|}{ Control Patients $(N=17)$} & \multirow{2}{*}{$\begin{array}{l}\mathrm{P} \text { Value (MBC } \\
\text { versus Control) }\end{array}$} \\
\hline & Positive, $n$ (\%) & Indeterminate, $n(\%)$ & Negative, $n(\%)$ & Positive, $n$ (\%) & Negative (\%) & \\
\hline ER & $62(95.4)$ & 0 & $3(4.6)$ & $15(88.2)$ & $2(11.7)$ & NS \\
\hline PR & $51(78.5)$ & 0 & $14(21.5)$ & $15(88.2)$ & $2(11.7)$ & NS \\
\hline MiB1 (Mean, 10.6\%) & $>10.6 \%, 19(29.2)$ & & $<10.6 \%, 46(70.7)$ & $>10.6 \%, 0$ & $<10.6 \%, 17(100)$ & 0.0088 \\
\hline P53 & $10(15.4)$ & $24(36.9)$ & $31(47.7)$ & $1(6)$ & $16(94.1)$ & 0.0087 \\
\hline Her2-neu & $6(9.2)$ & $3(4.6)$ & $56(86.2)$ & $0(0)$ & $17(100)$ & 0.10 \\
\hline
\end{tabular}

The tumors had a statistically significant higher expressions of MiB1, p53, and Her2-neu compared with the control samples.

The positivity rate is expressed as the absolute number and percentage in male breast tumors and gynecomastia. MiB1 is rated based on the number of patients above or below the average percentage positive in the tumor sample $(>10.6 \%$ or $<10.6 \%)$. P53 was rated based on degree of positive nuclear staining; positive was strong, uniform nuclear staining; indeterminate was weak or focal staining; negative was no staining. Her2-Neu positive was at least $2+$ in cytoplasmic staining according to the FDA-approved DAKO criteria. Her2-Neu- (negative) included indeterminate (1+), and negative (0). NS, not significant; MBC, male breast carcinoma.

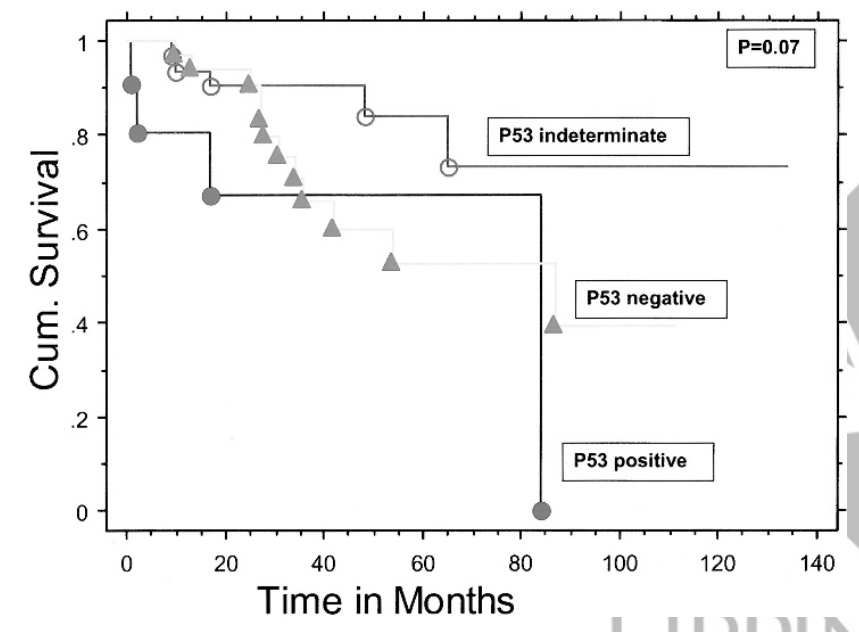

FIGURE 4. Disease-free survival in patients with p53-positive tumors was worse than patients with p53-negative or indeterminate tumors $(P$ $=.07)$. When stratified by stage, the difference became statistically significant $(P=.047)$.

tary service, occupational history, and other exposures. However, patients' similarities to the general population did exist in their age, geographical location, disease at presentation, and histological types. Therefore, many of the findings and conclusions apply not only to the veteran population, but to some degree, to the general male population.

The predominant histological type was infiltrating ductal carcinoma, although approximately one third of the carcinomas also had a component of DCIS. Tumor size, positive lymph nodes, tumor grade, presence of DCIS, or histological types did not contribute to prognosis in this cohort.

Many attempts have been made to study male breast carcinoma at the molecular and genetic levels. However, these studies have been limited to a small number of patients and have incomplete follow-up data. Hormone receptors in female breast carcinoma are well characterized. Positive expressions of ERs and PRs correlate with better survival and response to estrogen antagonists such as tamoxifen (15), regardless of tumor size, stage, and age. Other biomarkers useful in determining the prognosis of female breast cancer include tumor microvessel density, p53 expression, tumor size, and peritumoral lymphatic invasion (16) in node-negative tumors. Other prognostically important markers are increased Ki-67 activity associated with high mitotic index and high tumor grade (17), epidermal growth factor receptor, and tumor angiogenesis $(18,19)$.

In male breast carcinoma, several series reported a higher percentage of hormonal receptor positivity than female breast cancers. The ER-positive rate in our series $(95 \%)$ was higher than that seen in the female breast cancer or gynecomastia. However, many studies suggested that the expression of ER or PR in male breast carcinoma had no association with response to endocrine therapy or prognosis (20-22), whereas one series demonstrated a worse outcome (23). One earlier retrospective study suggested that adjuvant therapy in addition to surgery was associated with favorable survival (24). In our study, ER-positive tumors correlated with better survival than did ER-negative tumors in univariate analysis, but our ER-negative patients were few ( $n$ $=3$ ), and all died soon after the initial diagnosis. We found that hormonal therapy did not appear to benefit ER-positive patients. Neither chemotherapy nor radiation seemed to benefit survival in early- or late-stage patients. These controversial results may be due to the facts that (1) there was a selection bias because there was no consistent therapeutic regimen in hormone-positive or -negative patients and many received adjuvant therapy as a desperate attempt to improve survival; and (2) ER and PR may play different roles in their endocrine regulation of male breast versus female breast carcinoma.

In female breast cancer, p53 gene mutations and deletions are the most frequently observed singlegene alterations (25). p53 mutation inhibits cell death through apoptosis. The mutant p53 protein has a significantly longer half-life than the wildtype p53 protein and is detectable by immunohistochemical methods. Between 18 and $58 \%$ of male breast cancers were positive for p53 by immuno- 
TABLE 4. Summary of immunohistochemical stains versus grade, lymph node positivity, and early (Stage I/II)/late stages (Stage III/IV)

\begin{tabular}{|c|c|c|c|c|c|c|c|c|c|c|c|c|c|c|c|c|}
\hline & $\begin{array}{c}\mathrm{ER}+ \\
(n)\end{array}$ & $\begin{array}{c}\mathrm{ER}- \\
(n)\end{array}$ & $P$ & $\begin{array}{l}\mathrm{PR}+ \\
(n)\end{array}$ & $\begin{array}{c}\mathrm{PR}- \\
(n)\end{array}$ & $P$ & $\begin{array}{c}\operatorname{MiB} 1>10 \\
(n)\end{array}$ & $\begin{array}{c}\operatorname{MiB} 1<10 \\
(n)\end{array}$ & $P$ & $\begin{array}{l}\mathrm{P} 53+ \\
\quad(n)\end{array}$ & $\begin{array}{l}\mathrm{P} 53 \pm \\
\quad(n)\end{array}$ & $\begin{array}{l}\mathrm{P} 53- \\
(n)\end{array}$ & $P$ & $\begin{array}{l}\text { Her2+ } \\
\quad(n)\end{array}$ & $\begin{array}{l}\text { Her2- } \\
\quad(n)\end{array}$ & $P$ \\
\hline \multicolumn{17}{|l|}{ Grade } \\
\hline 1 & 8 & 0 & & 8 & 0 & & 2 & 6 & & 2 & 1 & 5 & & 0 & 8 & \\
\hline 2 & 27 & 1 & & 24 & 4 & & 6 & 22 & & 3 & 13 & 12 & & 3 & 25 & \\
\hline 3 & 23 & 2 & NS & 16 & 9 & 0.04 & 10 & 15 & NS & 4 & 8 & 13 & NS & 3 & 22 & NS \\
\hline \multicolumn{17}{|c|}{ Lymph nodes } \\
\hline+ & 20 & 1 & & 14 & 7 & & 6 & 15 & & 4 & 6 & 11 & & 5 & 14 & \\
\hline- & 29 & 1 & NS & 24 & 6 & NS & 8 & 22 & NS & 13 & 14 & 13 & NS & 1 & 29 & 0.08 \\
\hline \multicolumn{17}{|l|}{ Stage } \\
\hline I-II & 43 & 2 & & 36 & 9 & & 13 & 32 & & 6 & 20 & 19 & & 3 & 42 & \\
\hline III-IV & 16 & 1 & NS & 12 & 5 & NS & 6 & 11 & NS & 3 & 4 & 10 & NS & 3 & 14 & NS \\
\hline
\end{tabular}

Data reflect the actual cases in each category. $P$ values are stated for each categorical comparison using $\chi^{2}$ analysis. NS, not statistically significant.

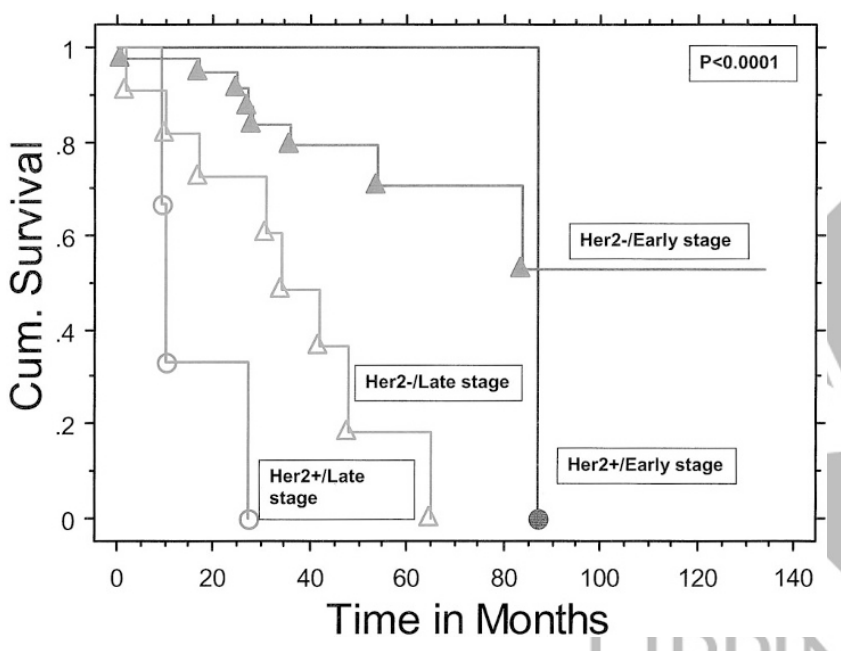

FIGURE 5. Her2 versus stage in disease-free survival. Her2-neu-positive and -negative tumors were stratified by patient's clinical stage, early (I/II) versus late (III/IV). In both early and late stages, patients with Her2 + tumors had a worse disease-free survival $(P$ $<.0001)$.

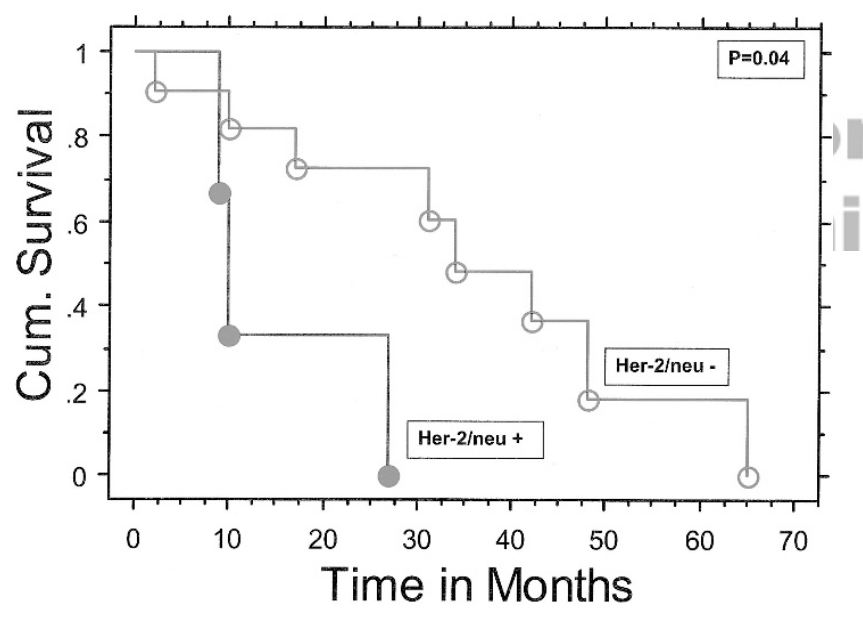

FIGURE 6. In Stage III/IV patients, Her2 positive (+) tumors had a shorter disease-free survival versus Her2 negative $(-)$ tumors $(P=.04)$.

histochemistry $(21,22,26)$ in three study series of $17-41$ patients, suggesting that male breast carcinoma contained abnormally expressed, mutant p53 protein. These studies showed a statistical trend (but one not statistically significant, because of small sample size) toward poorer outcome and larger tumor size. Positive p53 mutation (15.4\%) in our series had a statistically significant shorter disease-free survival when stratified by stage only, which confirmed the previous trend.

The monoclonal antibody MiB1 (Ki-67), a measurement of cell proliferative activity, was identified as a potential prognostic marker in breast carcinoma in which a higher percentage correlated with increased tumor mitotic index and tumor grade (17). Previous studies showed that $20-40 \%$ of male breast carcinomas were positive for $\operatorname{MiB1}(20,21)$. These carcinomas had weak to strong associations between high MiB1 scores and androgen receptor negativity and worse prognosis (20). Our mean MiB1 percentage was $10.6 \%$, higher than that of gynecomastia $(3.5 \%)$. However, higher MiB1 did not correlate with lymph node metastasis, tumor grade, stage, or disease-free survival.

The proto-oncogene Her2-neu (c-erbB-2), localized to chromosome 17q21, encodes a transmembrane tyrosine kinase growth factor receptor. Her2/neu shares considerable homology with the epidermal growth factor receptor. Her2-neu gene amplification has been associated with the development of breast cancer in animal models and is seen in $10-34 \%$ of breast carcinomas (27). One practical method in assessing Her2-neu status in routine clinical setting is by immunohistochemistry, although fluorescence in situ hybridization (FISH) for Her2-neu gene amplification may offer more information on disease prognosis and predicting treatment response $(28,29)$. On immunohistochemistry, a distinct, membranous staining pattern is seen when the receptor is present. Numerous studies of Her2-neu gene and protein expression correlate with poor prognosis in female breast cancers, especially in node-positive patients (30), but few data are available in the male counterpart. A few studies with limited numbers of patients show that Her2-neu protein overexpression as measured by immunohistochemistry is present 
in $1.7-45 \%$ of male breast carcinoma patients, but survival analysis is limited by small sample size (21, 22, 31-33) and by no clear association with survival. A recent breast cancer therapy directed against the Her2-neu (anti-Her2 antibodies-Herceptin, generic name trastuzamab) is undergoing clinical trial and shows potential benefit in the treatment of metastatic disease, either as a single agent or in combination with other chemotherapy (34). No clinical trials to date have applied anti-Her2 antibodies for the treatment of male breast carcinoma.

Her2-neu overexpression was low in our series (9\%) compared with other studies, and this may be due to a more stringent criteria for determining a positive expression, but positive Her2-neu was associated with worse survival in multivariate analysis on Her2neu and stage $(P=.0001)$, and Her2-neu positivity was seen more often in tumors with metastatic lymph nodes $(P=.08)$. Clinical trials for anti-Her2-neu may be beneficial in lymph node positive, late-stage, and Her2-neu-positive patients.

In conclusion, male breast carcinoma in the veteran population is a disease of older men and presents approximately 10 years later than does female breast cancer. Although the disease occurs later in life of men who frequently have many other medical illnesses, these patients do have a worse disease-free survival than do comparable men of similar age and medical background but with benign breast mass such as gynecomastia.

We evaluated five potential prognostic markers in the study of male breast carcinoma in this VA cohort. We found that advanced American Joint Committee on Cancer stage and positive Her2-Neu associated with Stage III/IV tumors were the only independent prognostic factors affecting adverse survival. The presence of ER, PR, or higher MiB1 percentage did not appear to be of significant prognostic value or helpful in guiding treatment. p53-positive tumors may be associated with worse disease-free survival. Positive Her2-neu expression was associated with higher number of positive lymph nodes and worse diseasefree survival. Anti-Her2-neu treatment may be of benefit in these patients. Surgery alone seemed to be sufficient treatment for the majority of the patients. Chemotherapy or radiation did not appear to add survival benefit in these patients. However, prospectively randomized, multicenter clinical trials using these markers to stratify male breast cancer patients are needed to confirm these findings.

Acknowledgments: The authors acknowledge Steven Claunch for his technical expertise in immunohistochemistry. The authors are grateful to the contributing VA medical centers (VAMCs) for their cases: Alexandria VAMC, Alexandria, LA; Alvin C. York VAMC, Murfreesboro, TN; Ann Arbor VAMC, Ann
Arbor, MI; Augusta VAMC, Augusta, GA; Biloxi VAMC, Biloxi, MS; Boise VAMC, Boise, ID; Boston VAMC, Boston, MA; Brooklyn VAMC, Brooklyn, NY; VA Medical Center, Fayetteville, Arkansas; Hunter Homes McGuire VAMC, Richmond, VA; G. V. Sonny Montgomery VAMC, Jackson, MS; Lexington VAMC, Lexington, KY; Clement J. Zablocki VAMC, Milwaukee, WI; New Orleans VAMC, New Orleans, LA; Portland VAMC, Portland, OR; VA Roseburg Health Care System, Roseburg, OR; Salem VAMC, Salem, VA; VA San Diego Health Care System, San Diego, CA; San Juan VAMC, San Juan, PR; Overton Brooks VA Medical Center, Shreveport, Louisiana; St. Louis VAMC, St. Louis, MO

\section{REFERENCES}

1. SEER. Cancer incidence public-use database (CD-ROM). Bethesda, MD; 1997.

2. Carmalt H, Mann L, Kennedy C, Fletcher J, Gillett D. Carcinoma of the male breast: a review and recommendations for management. Aust N Z J Surg 1998;68:712-5.

3. Williams W, Powers M, Wagman L. Cancer of the male breast: a review. J Natl Med Assoc 1996;88:439-43.

4. Yildirim E, Berberoglu U. Male breast cancer: a 22-year experience. Eur J Surg Oncol 1998;24:548-52.

5. Ravandi-Kashani F, Hayes T. Male breast cancer: a review of the literature. Eur J Cancer 1998;34:1341-7.

6. Tukel S, Ozcan H. Mammography in men with breast cancer: review of the mammographic findings in five cases. Australasian Radiol 1996;40:387-90.

7. Clark J, Nguyen P, Jaszcz W, Jatoi W, Niehans G. Prognostic variables in male breast cancer. Am Surg 2000;66:502-11.

8. Elston EW, Ellis IO. Pathological prognostic factors in breast cancer. I. The value of histological grade in breast cancer: experience from a large study with long-term follow-up. Histopathology 1991;19:403-10.

9. Jacobs T, Gown A, Yaziji H, Barnes M, Schnitt S. Specificity of HercepTest in determining HER-2/neu status of breast cancers using the United States Food and Drug Administrationapproved scoring system. J Clin Oncol 1999;17:1983.

10. Rosenblatt K, Thomas D, Jimenez L. The relationship between diet and breast cancer in men (United States). Cancer Causes Control 1999;10:107-13.

11. Hsing A, McLaughlin J, Cocco P, Chien H, Fraumeni J. Risk factors for male breast cancer (United States). Cancer Causes Control 1998;9:269-75.

12. Donegan W, Redlich P, Lang P, Gall M. Carcinoma of the breast in males. Cancer 1998;83:498-509.

13. Hill A, Yagmur Y, Tran K, Bolton J, Robson M, Borgen P. Male breast carcinoma and family history: an analysis of 142 patients. Cancer 1999;86:821.

14. Wick M, Sayadi H, Ritter J, Hill A, Reddy V, Gattuso P. Low-stage carcinoma of the male breast. Am J Clin Pathol 1999;111:59-69.

15. Shapiro C, Recht A. Side effects of adjuvant treatment of breast cancer. N Engl J Med 2001;344:1997-2008.

16. Gasparini G, Weidner N, Bevilacqua P, Maluta S, Palma P, Caffo O, et al. Tumor microvessel density, p53 expression, tumor size, and peritumoral lymphatic vessel invasion are relevant prognostic markers in node-negative breast cancer. J Clin Oncol 1994;12:454-66.

17. Weidner N, Moore D, Vartanian R. Correlation of Ki-67 antigen expression with mitotic figure index and tumor grade 
in breast carcinomas using the novel "paraffin"-reactive MiB1 antibody. Hum Pathol 1994;25:337-42.

18. Weidner N, Gasparini G. Determination of epidermal growth factor receptor provides additional prognostic information to measuring tumor angiogenesis in breast carcinoma patients. Breast Cancer Res Treat 1994;29:97-107.

19. Weidner N, Folkman J, Pozza F, Bevilacqua P, Allred E, Moore D, et al. Tumor angiogenesis: a new significant and independent prognostic indicator in early-stage breast carcinoma. J Natl Cancer Inst 1992;84:1875-87.

20. Margaria A, Chiusa L, Candelaresi G, Canton O. Androgen receptor expression in male breast carcinoma: lack of clinicopathological association. Br J Cancer 1999;79:959-64.

21. Willsher P, Leach I, Ellis I, Bell J, Elston C, Bourke J, et al. Male breast cancer: pathological and immunohistochemical features. Anticancer Res 1997;17:2335-8.

22. Joshi M, Lee A, Loda M, et al. Male breast carcinoma: an evaluation of prognostic factors contributing to a poorer outcome. Cancer 1996;77:490-8.

23. Bruce D, Heys S, Payne S, Miller I, Eremin O. Male breast cancer: clinico-pathological features, immunocytochemical characteristics and prognosis. Eur J Surg Oncol 1996;22:42-6.

24. Margaria E, Chiusa L, Ferrari L, Canton OD, Pich A. Therapy and survival in male breast carcinoma: a retrospective analysis of 50 cases. Oncol Rep 2000;7:1035-9.

25. Runnebaum I, Nagarajan M, Bowman M, Soto D, Sukumar S. Mutations in p53 as potential molecular markers for human breast cancer. Proc Natl Acad Sci U S A 1991;88:10657-61.
26. Anelli A, Anelli T, Youngson B, Rosen P, Borgen P. Mutations of the p53 gene in male breast cancer. Cancer 1995;75: 2233-8.

27. Press M, Cordon-Cardo C, Slamon D. Expression of the HER-2/neu proto-oncogene in normal human adult and fetal tissues. Oncogene 1990;5:953-62.

28. Schnitt S. Breast cancer in the 21st century: neu opportunities and neu challenges. Mod Pathol 2001;14:213-8.

29. Jimenez R, Wallis T, Tabasczka P, Visscher D. Determination of Her-2/Neu status in breast carcinoma: comparative analysis of immunohistochemistry and fluorescent in situ hybridization. Mod Pathol 2000;13:37-45.

30. Lee A, Wiley B, Loda M, et al. DNA ploidy, proliferation and new-oncogene protein overexpression in breast carcinoma. Mod Pathol 1992;5:61-7.

31. Pich A, Margaria E, Chiusa L. Oncogenes and male breast carcinoma: c-erB-2 and p53 coexpression predicts a poor survival. J Clin Oncol 2000;18:2948-65.

32. Shpitz B, Bomstein Y, Sternberg A, Klein E, Liverant S, Groisman G, et al. Angiogenesis, p53, c-erbB2 immunoreactivity and clinicopathological features in male breast cancer. J Surg Oncol 2000;75:252-7.

33. Bloom K, Gorvil H, Gattuso P, Reddy V, Francescatti D. Status of HER-2 in male and female breast carcinoma. Am J Surg 2001;182:389-92.

34. Ross J, Fletcher J. The Her-2/neu oncogene in breast cancer: prognostic factor, predictive factor, and target for therapy. Stem Cells (Dayt) 1998;16:413-28.
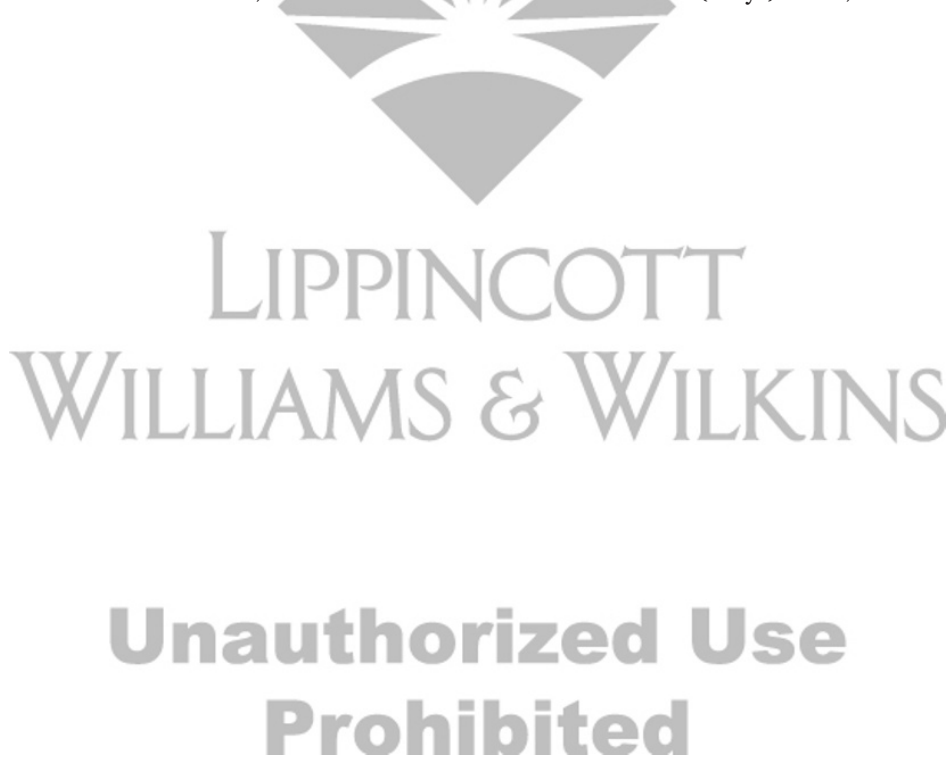